\title{
An Investigation of the Structure Underlying Irreducible Devisors
}

\author{
Hilary Smallwood \\ Fort Lewis College \\ 1000 Rim Drive \\ Durango, Colorado 81301 USA \\ Drew Swartz \\ Department of Mathematical Sciences \\ Indiana University-Purdue University Fort Wayne \\ 2101 East Coliseum Avenue \\ Fort Wayne, Indiana 46805 USA
}

Received: August 8, 2008

Accepted: October 13, 2008

\begin{abstract}
In previous literature Coykendall \& Maney, as well as Axtell \& Stickles, have discussed the concept of irreducible divisor graphs of elements in domains and ring with zero-divisors respectively, with two different definitions. In this paper we seek to look at the irreducible divisor graphs of ring elements under a hybrid definition of the two previous ones-in hopes that this graph will reveal structure concerning irreducible divisors in rings with zero-divisors. We also compare the three graphs and examine in what respects they are related. Other graph-theoretic properties of this graph will also be discussed.
\end{abstract}

\section{INTRODUCTION}

Throughout this paper we let $R$ denote a commutative ring with identity. For any ring $R$, we let $R^{*}=R \backslash\{0\}$. An element a $\in R$ is a zero-divisor if there exists a nonzero element $\mathrm{b} \in R$ such that $a b=0$. We define $Z(R)$ to be the set of zero divisors of $R$. An element $u \in R$ is a unit if there exists a $v \in R$ such that $u v=1$. We define $U(R)$ to be the set of units of $R$. For $x \in R \backslash U(R)$, $\mathrm{x}$ is irreducible if whenever $x=a b$, then $(x)=(a)$ or $(x)=(b)$, that is to say $x$ is either associate to $a$ or $b$, which will be denoted as $x \sim a$ or $x \sim b$. The set of irreducible elements of $R$ will be denoted as $\operatorname{Irr}(R)$; for $\mathrm{R}$ a domain this allows $0 \in \operatorname{Irr}(R)$. We will further restrict ourselves to $\overline{\operatorname{Irr}}(R)$, the set containing only associate class representatives for each given nonzero irreducible. Similarly we will let $\overline{\operatorname{Irr}}(x)$ denote the associate class representatives of irreducible divisors of a specific element $x$ $\in R \backslash U(R)$. A ring $R$ is said to be atomic if each nonzero nonunit can be factored into a product of irreducible elements. Throughout the paper, we will frequently restrict ourselves to atomic rings. A ring $R$ is a Unique Factorization Ring (UFR) if whenever $a_{1} a_{2} \ldots a_{s}$ and $b_{1} b_{2} \ldots b_{\mathrm{t}}$ are two factorizations of a given $x \in R^{*} U(R)$, with each $a_{i}$ and each $b_{\mathrm{j}}$ irreducible, then the following hold:

(1) $s=t$

(2) there exists a permutation $\sigma$ of $\{1,2, \ldots, t\}$ such that $a_{\mathrm{i}} \sim b_{\sigma(\mathrm{i})}$ for all $i \in$ $1,2, \ldots, t$

We define $G=(V, E)$ as a vertex set $V$ and an edge set $E$ consisting of ordered 
pairs of elements in $V$. Two elements $a, b \in$ YG] are said to be adjacent if there exists an edge between them. We will denote the edge between $a$ and $b$ by $a-b$.

We will at times abuse notation and use $a-b$ to mean that $a$ is adjacent to $b$. A path between two vertices $a_{1}, a_{n}$ is an ordered sequence, $\left\{a_{1}, a_{2}, \ldots, a_{n}\right\}$, of distinct vertices $G$ such that $a_{i}-a_{i+1}$. If $\left\{a_{1}, a_{2}, \ldots, a_{n}\right\}$ is a path in $G$ from the vertex $a_{1}$ to the vertex $a_{n}$, then the length of this path is $n-1$. The distance between vertices $a$ and $b$ in $G$, denoted $d(a, b)$, is the length of the shortest path between $a$ and $b$. If no path exists between two vertices $a$ and $b$, then we say that $d(a, b)=\infty$. The diameter of a graph $G$, denoted $\operatorname{diam}[G]$, is $\max \{d(a, b) \mid a, b \in V G]\}$. A vertex $a$ is said to be looped if there exists an edge from $a$ to itself. The graph $G$ is said to have a cycle if there exists a nontrivial path from vertex $a_{1}$ to itself with no repeated edges; that is, an ordered sequence $\left\{a_{1}, a_{2}, \ldots, a_{k}, a_{1}\right\}$, where $a_{i}-a_{i+1}$ for $1 \leq i \leq k-1$ and $a_{k}-a_{1}$. If a cycle exists in $G$ then we $g[G]=\infty$. A graph is said to be connected if there exists a path between any two distinct vertices. Furthermore a graph $G$ is complete if every vertex is adjacent to every other vertex; that is $G$ is connected and $\operatorname{diam}[G]=1$.

In [1] D. Anderson and P. Livingston introduced the zero-divisor graph $\Gamma(R)=$ $(V, E)$ for $R$, a commutative ring with identity. In this graph, the vertex $V$ consists of all nonzero zero-divisors of $R$ and there is an edge between to zero-divisors $a$ and $b$ if and only if $a b=0$ in $R$. Much research has been done regarding the zero-divisor graph and its properties. In [1], it was found that $\Gamma(R)$ is remarkably well structured. For example, if $R$ is a commutative ring, the $\operatorname{diam}[\Gamma(R)] \leq$ 3. Further, if $\Gamma(R)$ contains a cycle, the $g[\Gamma(R)] \leq 4$.

Irreducible divisor graphs were introduced by Coykendall and Maney [7] to generalize the zero-divisor graph to factorizations on nonzero elements in atomic domains. Given a domain $D$ and $x \in D \backslash U(D)$ such that $x$ can be factored into irreducible, they define the irreducible divisor graph, denoted $G(x)$, as $G(x)=(V, E)$, where $V=$
$\overline{\operatorname{Irr}}(x)$ and $y_{1}-y_{2} \in E$ if and only if $y_{1} y_{2} \mid x$. Using this definition they obtained some very nice results, such as the equivalence of the following statements for an atomic domain $D$ : 1) $D$ is a UFD; 2) $G(x)$ is complete for each $x \in D^{*} \backslash U(D)$; 3) $G(x)$ is connected for each $x \in D^{*} \backslash U(D)$.

Following Coykendall and Maney [7], Axtell and Stickles [4] further generalize the irreducible divisor graph to commutative rings with zero divisors. Before we define this alternate graph, let us first define a Ufactorization which will be an essential tool when factoring rings with zero divisors. Given $x \in R \backslash(\mathrm{R})$, a $U$-factorization of $x$ (as defined in [4]) is given by $x=$ $a_{1} a_{2} \ldots a_{\mathrm{m}}\left[b_{1} b_{2} \ldots b_{\mathrm{n}}\right]$, where the following hold:

(i) $a_{\mathrm{i}}, b_{\mathrm{j}} \in R \backslash U(R)$ for all $i$ and $j$

(ii) $x=a_{1} a_{2} \ldots b_{1} b_{2} \ldots b_{n}$

(iii) $(x)=\left(b_{1} b_{2} \ldots b_{\mathrm{n}}\right)$

(iv) $(x) \neq\left(b_{1} b_{2} \ldots b_{j} \ldots b_{n}\right)$ for $1 \leq j \leq n$

(v) $(x)=\left(a_{i} b_{1} b_{2} \ldots b_{n}\right)$ for $1 \leq \mathrm{I} \leq \mathrm{m}$

We call the $b_{j}$ 's essential divisors of $x$, and the $a_{i}$ 's inessential divisors of $x$. For notation sake let $\bar{a}$ denote the product of inessential divisors $a_{1} a_{2} \ldots a_{\mathrm{m}}$, and let $\bar{b}$ denote the product of essential divisors $b_{1} b_{2} \ldots b_{n}$.

Proposition 1 (Proposition 4.1 in [3]). Any factorization of $r \in R$ can be rearranged to form a U-factorization of $r$.

A ring $R$ is $U$-atomic if each nonzero, nonunit of $R$ has a U-factorization in which all the essential divisors are irreducible. Note that an atomic ring is also U-atomic. Furthermore, a ring $R$ is a $U$ Unique Factorization Ring (U-UFR) if whenever $\bar{m}\left[a_{1} a_{2} \ldots a_{\mathrm{s}}\right]$ and $\bar{n}\left[b_{1} b_{2} \ldots b_{\mathrm{t}}\right]$ are two $U$-factorizations of a given $x \in R^{*} \backslash U(R)$, with each $a_{\mathrm{i}}$ and each $b_{\mathrm{j}}$ irreducible, then the following hold:

(1) $s=t$

(2) there exists a permutation $\sigma$ of

$\{1,2, \ldots, t\}$ such that $a_{1} \sim b_{\sigma()}$ for all $i \in$ $\{1,2, \ldots t\}$.

For $x \in R \backslash(R)$, Axtell and Stickles [4] define the $U$-irreducible divisor graph, $G_{u}(x)=(V, E)$, by $V=\{y \in \operatorname{Irr}(R) \mid y$ is an essential divisor of $x\}$, and $y_{1}-y_{2} \in E$ if and only if $y_{1}$ and $y_{2}$ are essential divisors in the same U-factorization of $x$. This definition eliminates trivial factorizations of certain idempotent elements. For example, in $\mathbb{Z}_{6}$ 
we have $3=3 \cdot 3=3^{n}$ for all $n \in \mathbb{N}$, but the corresponding U-factorization, $3=3^{n}[3]$, yields only one essential divisor. While Ufactorization provides a useful way of factoring, $G_{u}(x)$ is more restrictive in the forming of edges. Thus some structure is lost when going from $G(x)$ to $G_{u}(x)$.

In our research we combine the two previous definitions of the irreducible divisor graph to create a hybrid definition in which we take the vertex set from the more restrictive U-irreducible divisor graph, but the edge formations from the original irreducible divisor graph.

Definition 1. Let $R$ be an atomic, commutative ring with unity. Let $x \in R \cup(R)$. Define the hybrid irreducible divisor graph of $x, \gamma(x)=(V, E)$, with $V=\{y \in \operatorname{Irr}(R) \mid y$ is an essential divisor $x\}$, and where $y_{1}-y_{2} \in E$ if and only if $y_{1} y_{2} \mid x$.

Henceforth we will be exploring the structure underlying irreducible divisors by comparing the three graphs, $G(x), G_{u}(x), \gamma(x)$, and examining in what respects they are related. Other graph-theoretic properties of $\gamma(x)$ will also be discussed.

\section{THE GRAPH $\gamma(0)$}

When we are in a domain, the graph $\gamma(0)$, as well as the graphs $G(0)$ and $G_{u}(0)$, are trivial graphs consisting of one vertex, 0 (since 0 is irreducible in a domain). In a ring with zero divisors, these graphs have interesting properties. The irreducible divisor graphs of zero can be thought of as a modification to the zero-divisor graph, $\Gamma(R)$. We see in the following theorem that $\gamma(0)$ exhibits more graph structure than does $\Gamma(R)$.

Theorem 1. For any atomic ring $\mathrm{R}$, the graph $\gamma(0)$ is complete (and hence connected).

Proof. Let $a, b \in \mathrm{V}[\gamma(0)]$. If $a$ and $b$ appear in the same U-factorization of 0 , as either essential divisors, inessential divisors or both, then $a b \mid 0$, hence $a-b$ in $\gamma(0)$. If $a$ and $b$ appear only in different U-factorizations, there exists some $c \in R$ such that $a c=0$, which implies there is a U-factorization such that $0=b[a c]$. Then $a b \mid 0$ and hence $a-b$ in $\gamma(0)$. Thus $\gamma(0)$ is complete (and hence connected).

A similar proof for the completeness of $G(0)$ can be found in [4]. Though $\gamma(0)$ and $G(0)$ are always complete, Example 3.2 in [4] shows that $G_{u}(0)$ is not necessarily complete. The following is a similar example with illustrates this fact.

Example 1. Let $R=\mathbb{Z}_{5}[x, y, z] /(x y, y z)$. Since $0=[x y]=[y z]$ we see $x$ and $z$ both appear as essential divisors in some $U$-factorization of 0 . Consider $0=x z \cdot f(x, y, z)$. By Proposition 1 this can be rearranged into a $U$-factorization. Now say $f(x, y, z) \in \operatorname{ann}(x z)$ $=(y)$; therefore, $f(x, y, z)=y \cdot m$ for some $m \in$ $R$. So $0=x z \cdot f(x, y, z)=x z \cdot y m$, which can only be rearranged into the following $U$ factorizations: $0=x m[y z]$ or $0=z m[x y]$ or 0 $=x z[y m]$. In any case, $x$ and $z$ never appear as essential divisors in the same $U$ factorization of 0 . Hence, $G_{u}(0)$ is not complete.

Even though the graph $G_{u}(0)$ is not incomplete, it is in fact connected as is shown in Proposition 3.4 of [4].

\section{COMPARING AND CONTRASTING} $\gamma(x), G(x)$, AND $_{u}(x)$

In [4] the authors posed various questions regarding the connectedness and completeness of the graphs $G(x)$ and $G_{u}(x)$. Here we extend those questions to the graph $\gamma(x)$, which will serve as a means for comparing $\gamma(x)$ to $G(x)$ and $G_{u}(x)$. Before we proceed, let us quickly provide the definition of a subgraph. Given a graph A, B is a subgraph of $\mathrm{A}$ if the following hold:

(1) $Y \mathrm{~B}] \subseteq V \mathrm{~A}]$

(2) $E[B] \subseteq E[A]$

(3) Whenever $a-b \in E[\mathrm{~B}]$, then $a, b \in$ VB].

Thus by construction it is evident that $G_{u}(x)$ is a subgroup of $\gamma(x)$, and $\gamma(x)$ is a subgroup of $G(x)$.

For any atomic ring $R$, we consider the following questions:

(1) If $R$ is a UFR, is $\gamma(x)$ connected (resp. complete) for every $x \in$ $R^{\star} \backslash \bigcup(R)$ ? 
(2) If $R$ is a U-UFR, is $\gamma(x)$ connected (resp. complete) for every $x \in$ $R^{\star} \backslash \cup(R)$ ?

(3) If $\gamma(x)$ is connected for every $x \in$ $R^{\star} \backslash U(R)$, is $R$ a UFR?

(4) If $\gamma(x)$ is connected for every $x \in$ $R^{\star} \backslash U(R)$, is $R$ a U-UFR?

(5) If $\gamma(x)$ is complete for every $x \in$ $R^{\star} \backslash U(R)$, is $R$ a UFR?

(6) If $\gamma(x)$ is complete for every $x \in$ $R^{\star} \backslash U(R)$, is $R$ a U-UFR?

(7) If $x \in R^{\star} \backslash U(R)$ and $G(x)$ is connected, is $\gamma(\mathrm{x})$ connected?

(8) If $x \in R^{\star} \backslash U(R)$ and $G_{u}(x)$ is connected, is $\gamma(x)$ connected?

(9) If $x \in R^{\star} \backslash U(R)$ and $G(x)$ is complete, is $\gamma(x)$ complete?

(10) If $x \in R^{\star} \backslash U(R)$ and $G_{u}(x)$ is complete, is $\gamma(x)$ complete?

(11) If $x \in R^{\star} \backslash U(R)$ and $\gamma(x)$ is connected, is $G(x)$ connected?

(12) If $x \in R^{\star} \backslash U(R)$ and $\gamma(x)$ is connected, is $G_{u}(x)$ connected?

(13) If $x \in R^{\star} \backslash U(R)$ and $\gamma(x)$ is complete, is $G(x)$ complete?

(14) If $x \in R^{\star} \backslash U(R)$ and $\gamma(x)$ is complete, is $G_{u}(x)$ complete?

While the answers to (1) and (2) are clearly yes, answers to the remaining questions are not quite as obvious. The following examples and theorems will provide answers for the majority of questions (3) - (14).

Lemma 1. Let $R$ be an atomic ring, $x \in$ $R \backslash \cup(R)$, and $a \in V \gamma(x)]$. If a appears as an inessential divisor in any $U$-factorization of $x$, then $a-v$ for all $v \in V \gamma(x)] \backslash\{a\}$.

Proof. First note that since $R$ is atomic, all divisors of $x$ appearing in the proof will be assumed to be irreducible. Since $a$ appears as an inessential divisor in some factorization of $x$, we have that $x=$ $a b_{1} b_{2} \ldots b_{n}\left[c_{1} c_{2} \ldots c_{m}\right]$ is a U-Factorization of $x$. Let $x=d_{1} d_{2} \ldots d_{j}\left[f_{1} f_{2} \ldots f_{h}\right]$ be an arbitrary $U$ factorization of $x$. Thus we have that $(x)=$ $\left(f_{1} f_{2} \ldots f_{h}\right)=\left(c_{1} c_{2} \ldots c_{m}\right)=a\left(f_{1} f_{2} \ldots f_{h}\right)$. Therefore, $a f_{i} \mid x$ for all $i$. Thus $a-v$ for all $v$ $\in V \gamma(x)] \backslash\{a\}$.

By a similar argument, we see that for an atomic ring $R, x \in R \backslash \cup(R)$, and $a \in$ $\bigvee G(x)$ ], if a ever appears as an inessential divisor in some $U$-factorization of $x$, then $a$ $v$ for all $v \in Y G(x)] \backslash\{a\}$.

Theorem 2. For any atomic ring $\mathrm{R}$ and $x \in$ $R^{\star} \backslash U(R), \gamma(x)$ is complete if and only if $G(x)$ is complete.

Proof $(\Rightarrow)$ Let $x \in R^{\star} \backslash U(R)$ with $a, b \in \operatorname{Irr}(x)=$ $\eta G(x)]$. Suppose $a, b \in V \gamma(x)]$. Since $\gamma(x)$ is complete, $a-b$ in $\gamma(x)$. Thus $a-b$ in $G(x)$ as well. If without loss of generality, $a, b \in$ $\eta G(x)] \backslash V \gamma(x)]$, then $a$ is never an essential divisor of $x$, and thus is always an inessential divisor. Thus by the note following Lemma 1, $a$ is connected to every other vertex in $G(x)$. Hence $G(x)$ is complete. $(\Leftarrow)$ For any $a, b \in V \gamma(x)], a, b \in$ $\eta G(x)]$, because $\bigvee \gamma(x)] \subseteq \bigvee G(x)]$. Since $G(x)$ is complete, $a$ is connected to every other vertex in $G(x)$, in particular $b$; implying $a b \mid x$, so $a-b$ in $\gamma(x)$ as well. Since $a$ and $b$ were arbitrarily, $\gamma(x)$ is complete.

Theorem 3. For any atomic ring $R$ and $x \in$ $R^{\star} \backslash U(R)$, if $G_{u}(x)$ is complete, then $\gamma(x)$ is complete.

Proof. Let $x \in R^{\star} \backslash U(R)$ with $\left.a, b \in \bigvee \gamma(x)\right]$. Observe that $\left.\bigvee \gamma(x)]=\bigvee G_{u}(x)\right]$. Since $\left.G_{u}(x)\right]$ is complete, we see that $a b \mid x$; hence, $a-b$ in $\gamma(x)$ as well. Since $a$ and $b$ were chosen arbitrarily, $\gamma(x)$ is complete.

Thus we see that if $G(x)$ or $G_{u}(x)$ is complete then $\gamma(x)$ is complete; providing affirmative answers to questions (9) and (10). In regards to question (13), Theorem 2 showed that the completeness of $\gamma(x)$ implies that $G(x)$ is complete; however the converse of Theorem 3, also appearing as question (14), is false, as the following example will show. However, before providing the example, we must introduce some new terms and a theorem concerning ring decompositions.

$$
\begin{aligned}
\text { Let } \mathrm{R} & \text { be } \mathrm{a} \text { ring such that } \\
R & \cong \prod_{\omega \in \Omega} R_{\omega}
\end{aligned}
$$

where $|\Omega| \geq 2$ and $R_{\omega}$ is a commutative ring with for all $\omega$ (note that $\Omega$ may be an 
infinite or finite indexing set). Let $\varphi$ denote the isomorphism between $\mathrm{R}$ and

$$
\prod_{\omega \in \Omega} R_{\omega}
$$

Given $x \in R$ we define $x$ to be an indecomposable element of $R$ if there exits exactly one nonunit component of $\varphi(x), x_{\omega}$, where $x_{\omega} \in R_{\omega} \backslash U\left(R_{\omega}\right)$. Now, before presenting the following theorem, we note that an element

$$
x \in \prod_{\omega \in \Omega} R_{\omega}
$$

where $|\Omega| \geq 2$, is irreducible if and only if there exists exactly one irreducible component of $x$, with all other components being units. That is, for

$$
x \in \prod_{\omega \in \Omega} R_{\omega}
$$

where $|\Omega| \geq 2$, there exists a $x_{\omega}$, such that $x_{\omega} \in \overline{\operatorname{Irr}}\left(R_{\omega}\right)$ for some $\omega \in \Omega$, and $x_{i} \in U\left(R_{i}\right)$ for all $i \neq \omega$ (Theorem 2.15 in [2]). Also, for the sake of presentation, we utilize tuples in the following theorem, which are assumed to contain a finite number of components. Even so, the theorem is still valid when the indexing set $\Omega$ is infinite, and the proof is analogous.

Lemma 2. Let $R$ be an atomic ring and

$$
\varphi: R \rightarrow \prod_{\omega \in \Omega} R_{\omega}
$$

be an isomorphism with $|\Omega| \geq 2$. Let $x \in R$ be an indecomposable element with $\varphi(x)=$ $\left(u_{1}, u_{2}, \ldots, u_{i-1}, x_{i}, u_{i+1} \ldots, u_{n}\right)$, where $x_{i} \in R_{i} \backslash U\left(R_{i}\right)$ and $u_{j} \in R_{j}$ for all $j \neq i$. Then $\cong \gamma\left(x_{i}\right)$, and

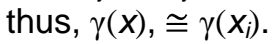

Proof. Let $\mathrm{V}\left[\gamma\left(x_{i}\right)\right]=\left\{b_{1}, b_{2}, b_{3}, \ldots, b_{n}\right\}$. Then any $\beta_{i} \in \mathrm{V}[\gamma(\varphi(x)]$ will be of the form $\left(u_{1}, u_{2}, \ldots u_{i-1}, \quad b_{i}, u_{i+1}, \ldots, u_{n}\right)$ for some $b_{i} \in$ $\mathrm{V}\left[\gamma\left(x_{i}\right)\right]$. Let $\Phi: \mathrm{V}\left[\gamma\left(x_{i}\right)\right] \rightarrow \mathrm{V}[\gamma(\varphi(x))]$, defined by $\Phi\left(b_{i}\right)=\left(1,1, \ldots, 1, b_{i}, 1, \ldots, 1\right)$. Notice that $b_{i}$ $\sim c_{i}$ if and only if $\left(1,1, \ldots, 1, b_{i}, 1, \ldots, 1\right) \sim$ $\left(1,1, \ldots, 1, b_{i}, 1, \ldots, 1\right) \sim\left(1,1, \ldots, 1, c_{i}, 1, \ldots, 1\right)$ since units are all in the same associate class as 1. Thus, associate classes are preserved under the mapping. Clearly, $\Phi$ is bijective and preserves edges between vertices, while creating no new edges; therefore $\Phi$ is an isomorphism between the two graphs. Clearly $\gamma(x) \cong \gamma(\varphi(x))$; therefore, we conclude that $\gamma(x) \cong \gamma\left(x_{i}\right)$.

We now present the following example which provides a negative answer to question (14).

Example 2. Let $R=Z_{5}[x, y, z] /(x y, y z)$ as in Example 1, and define $\mathrm{R} 1=\mathrm{Z} \times R$. Now consider $(1,0) \in R_{1}$. By Lemma 2, $\gamma(0) \cong$ $\gamma((1,0))$ and thus $\gamma((1,0))$ is complete since $\gamma(0)$ is complete. Similarly, Lemma 2 can be extended to accommodate $G_{u}(x)$, so we can see that $G_{u}(x) \cong G_{u}((1,0))$. However, it was shown in Example 1 that $G_{u}(0)$ is not complete. Thus by Lemma 2 it is clear $G_{u}((1,0))$ is not complete.

We now proceed to examine connectedness properties amongst the graphs. The following theorem will provide an affirmative answer to question (8).

Theorem 4. For any atomic ring $\mathrm{R}$ and $x \in$ $R^{\star} \backslash U(R)$, if $G_{U}(x)$ is connected then $\gamma(x)$ is connected.

Proof. Observe that $\left.\bigvee \gamma(x)]=\bigvee G_{u}(x)\right]$ and $E\left[G_{u}(x)\right] \subseteq E[\gamma(x)]$. The result clearly follows.

Theorem 5. For any atomic ring $R$ and $x \in$ $R^{\star} \backslash U(R)$. If $\gamma(x)$ is connected then $G(x)$ is connected.

Proof. Assume $\gamma(x)$ is connected. Let $a, b$ be vertices of $G(x)$. If $a$ and $b$ are essential divisors of $x$ (potentially in different $U$ factorizations), then there exists a path between them in $\gamma(x)$. This path is preserved in $G(x)$. Now, without loss of generality suppose that $a$ never appears as an essential divisor in any U-factorization of $x$. Since $a \in \operatorname{Irr}(x)$, $a$ must appear as an inessential divisor of $x$. Thus, by the statements following Lemma $1, a$ is connected to every other vertex in $G(x)$, and hence, a path exists from $a$ to $b$, in fact $a-$ b.

The above theorem answers (11) in the affirmative. The remaining questions regarding connectedness, (7) and (12), remain open. We now proceed to look at whether we can determine if $R$ is a UFR or a U-UFR based on the completeness or 
connectedness of $\gamma(x)$. The following example shows that a ring $R$ will not necessarily be UFR provided that $\gamma(x)$ is complete for all $x \in R^{\star} \backslash U(R)$.

Example 3. Consider the ring $\mathbb{Z}_{6}$. We need only consider the graphs $\gamma(2)$ and $\gamma(3)$ because $\operatorname{Irr}(R)=\{2,3\}$ (up to associates). Hence, we obtain the following graphs:
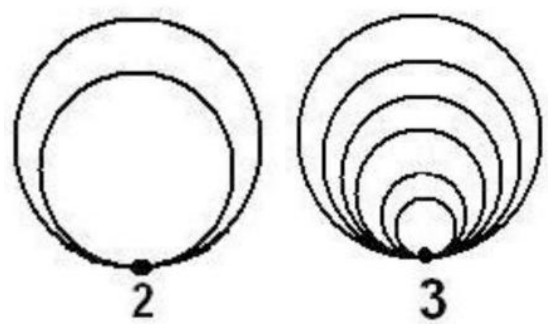

Both graphs are complete; however, $\mathrm{R}$ is certainly not a UFR since $2=2^{3}$, and $3=3.3$ $=3^{n}$ for any $n \in \mathbb{N}$.

The following example was originally given as Example 4.5 in [4], where the authors utilized it to show that a ring $R$ is not necessarily a U-UFR even when the graph $G_{u}(x)$ is complete for all $x \in R^{\star} \backslash U(R)$. The same example can be extended to $\gamma(x)$ to obtain a similar result.

Example 4. Let $R_{1}=\mathbb{Z}_{2}[y, z] /\left(y^{2}, z^{2}, y z\right)$. Consider the ring $R=\mathbb{Z}_{2} \times R_{1}$. It was shown in [4] the graph $G_{u}(x)$ is complete for all $x \in$ $R^{\star} \backslash \cup(R)$. By Theorem 3, $\gamma(x)$ is also complete for all $x \in R^{\star} \backslash U(R)$. However, when we consider $(1,0) \in R^{\star} \backslash U(R)$ we see that $(1,0)=[(1, y)(1, z)]$ and $(1,0)=$ $[(1, y+z)(1, y+z)]$ are two distinct $U$ factorizations of $(1,0)$. Hence, $R$ is not a $U$ UFR. Thus we see that a ring $R$ will not necessarily be a U-UFR even when the graph $\gamma(x)$ is complete for all $x \in R^{\star} \backslash U(R)$.

The two preceding examples provide negative answers to (5) and (6). Likewise, since complete implies connected, we have negative answers to (3) and (4).

Earlier it was noted that by construction, $G_{u}(x)$ is a subgraph of $\gamma(x)$, which is a subgraph of $G(x)$. We conclude this section by examining whether proper containments exist amongst the graphs. That is to say, does there exist a ring $R$, and an element $x \in R$ with $G_{u}(x), \gamma(x), G(x)$ pairwise distinct? Note that by pairwise distinct we mean $G_{u}(x) \neq \gamma(x), \gamma(x) \neq G_{u}(x)$, and $G_{u}(x) \neq G(x)$.

Example 5. Let $R=K[x, y, z] /(x y, y z)$ where $K$ is a finite field. Then define $R_{1}=\mathbb{Z} \times R$. Consider the element $(1,0) \in R_{1}$. Some irreducible factorizations of $(1,0)$ are as follows: $(1,0)=(1, x+y)(1, x)(1, y)=(1, y)(1, z)$ $=(1, x+z)(1, y)$. Thus we will only look at the subgraphs containing the vertices $(1, x+y)$, $(1, x),(1, y),(1, z),(1, x+z)$.

The edges in $G(x)$ are obvious from the given factorizations. Recalling Proposition 1, we know that any factorization of $(1$,$) can be arranged into$

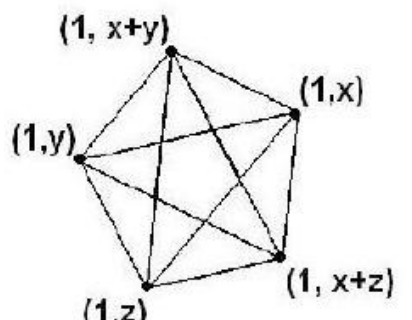

$(1,2)$

$\mathrm{G}((1,0))$

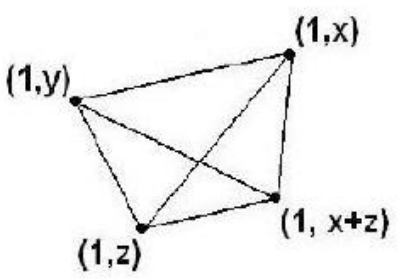

$\gamma((1,0))$

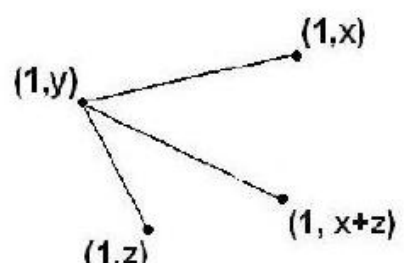

$(1,2)$

$\mathrm{G}_{\mathrm{u}}((\mathbf{1 , 0 )})$ a U-factorization. Thus $(1, x+y)$ is never an essential divisor of $(1,0)$. To illustrate this, suppose $(1, x+y)$ were an essential divisor.
Then $(1,0)=[(1, x+y)(1, f(x, y, z))]$. Hence $(1,0)=(1, x+y)(1, f(x, y, z))=(1, f(x, y, z) \cdot x+$ $f(x, y, z) \cdot y)$, which implies $f(x, y, z) \cdot x=$ 
$-f(x, y, z) \cdot y$. This will only happen when $f(x, y, z)=0=x y=y z$. Thus, $(1, x+y)$ will never be an essential divisor.

Since $(1, x+y)$ is never an essential divisor it is not in the vertex set of $\gamma((1,0))$. Hence a vertex is lost as well as all edges adjoined to it when going from $G((1,0))$ to $\gamma((1,0))$, so clearly $\gamma((1,0)) \neq G((1,0))$. Furthermore, we see in $G_{u}((1,0))$ that $(1,0)=$ $[(1, x+z)(1, y)]$ is a U-factorization where $(1, x+z)$ is an essential divisor of $(1,0)$. However, no two $(1, x+z),(1, x)$, or $(1, z)$ will ever appear together as essential divisors of $(1,0)$ in the same $U$-factorization. Thus the edges between them are lost when going from $\gamma((1,0))$ to $G_{u}((1,0))$, hence $G_{u}((1,0)) \neq$ $\gamma((1,0))$.

So we do in fact find that the graphs $G_{u}(1,0), \quad \gamma(1,0)$, and $G(1,0)$ are pairwise distinct. Thus in general, while $G_{u}(x)$ is a subgraph of $\gamma(x)$, which is a subgraph of $G(x)$, the graphs are distinct.

\section{REALIZABLE GRAPHS}

In literature such as [5] and [6], frequent references to complete bipartite and star graphs were made to the zerodivisor graph, $\Gamma(R)$ (as well as complete bipartite reducible and star-shape reducible graphs). In these papers it is shown that the zero-divisor graph $\Gamma(R)$ may be realized in these forms. We wish to explore whether the same holds true for the irreducible divisor graphs $\gamma(x), G_{u}(x)$, and $G(x)$.

Let us quickly provide definitions of these terms before proceeding. First, a graph is said to be complete bipartite if the set of vertices may be partitioned into two disjoint sets, $A$ and $B$, in which for all $a_{i} \in A$ and $b_{j} \in B, a_{i}-b_{j}$; and no edge is formed between $a_{i}$ and $a_{k}$ for all $a_{i}, a_{k} \in A$ (and all $b_{j}, b_{h} \in B$ ). As stated in [9], we denote a complete bipartite graph as $K_{m, n}$, where $|A|=m$ and $|B|=n$. Furthermore, a graph $\Phi$ is said to be complete bipartite reducible if there exists a complete bipartite subgraph $\Phi_{o}$ of $\Phi$ in which $\Phi_{o}$ is formed by removing only edges from $\Phi$. As in [9], we define a graph $\Phi$ to be a star graph if $\Phi$ is the complete bipartite graph $K_{1, n}$. That is, $\Phi$ may be partitioned into two disjoint sets, $A$ and $B$, with $A$ containing only one vertex. We call this single vertex the center vertex of the star graph. Also, $\Phi$ is said to be star- shape reducible if there exists a star subgraph $\Phi_{o}$ of $\Phi$ such that $\Phi_{o}$ is formed by removing only edges from $\Phi$.

Specifically for uses in this paper, we define a trivial star graph to be the graph $\Phi$ consisting of a single vertex, or two vertices with an edge joining them. We define a trivial complete bipartite graph similarly. For examples of these, consider the following graphs. First, $\gamma(6)$ in $\mathbb{Z}$ will contain two vertices, 2 and 3 , with $2-3$. Additionally for any prime $p \in \mathbb{Z}, \gamma(p)$ in $\mathbb{Z}$ will be the singleton graph consisting of the vertex $p$ (and no loops). However, the following theorems show that in a domain $\gamma(x)$ will never be realized as the non-trivial forms of these graphs. Observe that a nontrivial star graph would be of the form $K_{1, n}$ for $n \geq 2$; and a non-trivial complete bipartite graph would be of the form $K_{m, n}$, where, without loss of generality, $m \geq 1$ and $n \geq 2$.

Note that since we are restricted to domains in the following theorems, all divisors in a U-factorization are essential divisors. Hence, U-factorizations lose their significance. Thus, it is clear that in a domain, $G_{u}(x)=\gamma(x)=G(x)$.

Theorem 6. Let $D$ be an atomic domain and $x \in D^{*} \backslash U(D)$, then $\gamma(x)$ is not realizable as a non-trivial star graph.

Proof. Suppose $\gamma(x)$ is a non-trivial star graph. Let $a$ denote the center vertex of $\gamma(x)$. Let all other vertices in the graph be demoted $\delta_{1}, \delta_{2}, \delta_{3}, \ldots, \delta_{n}$. Since a must appear in every factorization of $x$ (since it is the center vertex), we have that:

$$
x=a \delta_{1}=a \delta_{2}=a \delta_{3}=\ldots=a \delta_{n}
$$

Hence, it is clear that $\frac{x}{a}=\delta_{1}=\delta_{2}=\delta_{3}=\ldots=$ $\delta_{n}$. Thus, $\delta_{1}=\delta_{2}=\delta_{3}=\ldots=\delta_{n}$. Thus, $\delta_{1}, \delta_{2}$, $\delta_{3}, \ldots, \delta_{n}$ are not distinct vertices in $\gamma(x)$. Hence, we see that $\bigvee \gamma(x)]=\{a, \delta\}$ and $a-\delta$ is the sole edge. Thus, $\gamma(x)$ violates the definition of a no-trivial star graph.

Whether $G_{u}(x), \gamma(x)$, or $G(x)$ may be realized as a non-trivial star graph in a ring with zero-divisors remains an open question. While $\gamma(x)$ will never take the form of a nontrivial star graph when $x$ is an element of a domain, Lemma 1 leads us to the conclusion that $\gamma(x)$ may be realized as a star-shape reducible graph (particularly in rings with 
zero-divisors). Let us elaborate slightly on this fact. Suppose we have an atomic ring $R, x \in R^{*} \cup(R)$, and $\left.a \in Y \gamma(x)\right]$ such that $a$ appears as an inessential divisor of $x$ in some U-factorization. Then by Lemma 1 , we may conclude that $a-b$ for all $b \in$ $\bigvee \gamma(x)] \backslash\{a\}$. Thus, $\bigvee \gamma(x)]$ can be broken up into two disjoint sets $A$ and $B$; with $a$ being the sole vertex contained in $A$ and all other vertices contained in $B$; and an edge connecting $a$ and $b$ for all $b \in B$; thereby giving us a star-shape reducible graph. (Note that there will be edges formed among vertices in $B$ ).

Theorem 7. Let $D$ be an atomic domain and $x \in D^{\star} \cup(D)$, then $\gamma(x)$ will not be realized as a non-trivial complete bipartite graph.

Proof. Suppose that $\gamma(x)$ is a non-trivial complete bipartite graph. Theorem 6 shows us that $\gamma(x)$ may not be realized as $K_{1, n}$ for any $n \geq 2$. Now, the vertices of $\gamma(x)$ may be partitioned into two disjoint sets, $A_{1}$ and $A_{2}$, with $\left|A_{1}\right|,\left|A_{2}\right| \geq 2$. Let $A_{1}=\left\{\alpha_{1}, \alpha_{2}, \ldots, \alpha_{n}\right\}$, $A_{2}=\left\{\varepsilon_{1}, \varepsilon_{2}, \ldots, \varepsilon_{m}\right\}$.

Since $\gamma(x)$ is complete bipartite by hypothesis, we have that there exist two distinct U-factorizations of $x$ of the following form: $x=\alpha_{i} \varepsilon_{j}$ and $x=\alpha_{i} \varepsilon_{k}$, with $j \neq k$. Thus we have that $\frac{x}{\alpha_{i}}=\varepsilon_{\mathrm{i}}=\varepsilon_{\mathrm{k}}$. Hence $\varepsilon_{\mathrm{i}}=\varepsilon_{k}$; which implies that $\varepsilon_{1}, \varepsilon_{2}, \ldots, \varepsilon_{m}$ are not distinct vertices. Thus $\left|A_{2}\right|=1$. This contradicts $\gamma(x)$ being a non-trivial complete bipartite graph.

Whether $G_{u}(x), \gamma(x)$, or $G(x)$ may be realized as non-trivial complete bipartite in a ring with zero-divisors again remains an open question. Thus, for the moment, we see that there are many unanswered and/or unexamined questions concerning the structure of $\gamma(x)$, especially when considering rings with zero-divisors. In future research, the authors will further examine the structure of $\gamma(x)$, particularly focusing on the diameter and girth of the graph.

\section{ACKNOWLEDGEMENTS}

The authors wish to acknowledge and thank the following persons/institutions for their aid throughout the production of this paper:

- The Wabash Summer Institute in Mathematics

- Prof. Michael Axtell

- Prof. Joe Stickles

- The National Science Foundation

\section{REFERENCES}

1. Anderson, D. and Livingston, P., The zero-divisor graph of a commutative ring, J. of Algebra, 217 (1999) pp. 434447.

2. Anderson, D.D. and Valdes-Leon, D., Factorization in commutative rings with zero-divisors, Rocky Mountain J. Math, 26 (1996), pp. 439-480.

3. Agargun, A.G., Anderson, D.D., and Valdes-Leon, S., Factorization in commutative rings with zero-divisors, III, Rocky Mountain J. Math., 31 (2001), pp. 1-21.

4. Axtell, M. and Stickles, J., Irreducible divisor graphs in commutative rings with zero-divisors, Comm. Algebra, 36(5) (2006) pp. 1883-1893.

5. Axtell, M., Stickles, and J. Trampbachls, P., Zero-divisors ideals and realizable zero-divisor graphs, submitted.

6. Axtell, M., Stickles, J., and Warfel, J., Zero-divisor graphs of direct products of commutative rings, Houston J. Math., 32(4) (2006), pp. 985-994.

7. Coykendall, J. and Maney, J., Irreducible divisor graphs, Comm. Algebra, 35(3) (2007) pp. 885-895.

\section{Wabash College} A LIBERAL ARTS COLLEGE FOR MEN

\section{Wabash Summer Institute in Mathematics (WSIM)}

The Wabash Summer Institute in Mathematics offers 12 undergraduates an excellent opportunity to conduct mathematical research with top faculty on the campus of a private college. See http://www.wabash.edu/academics/math/ 


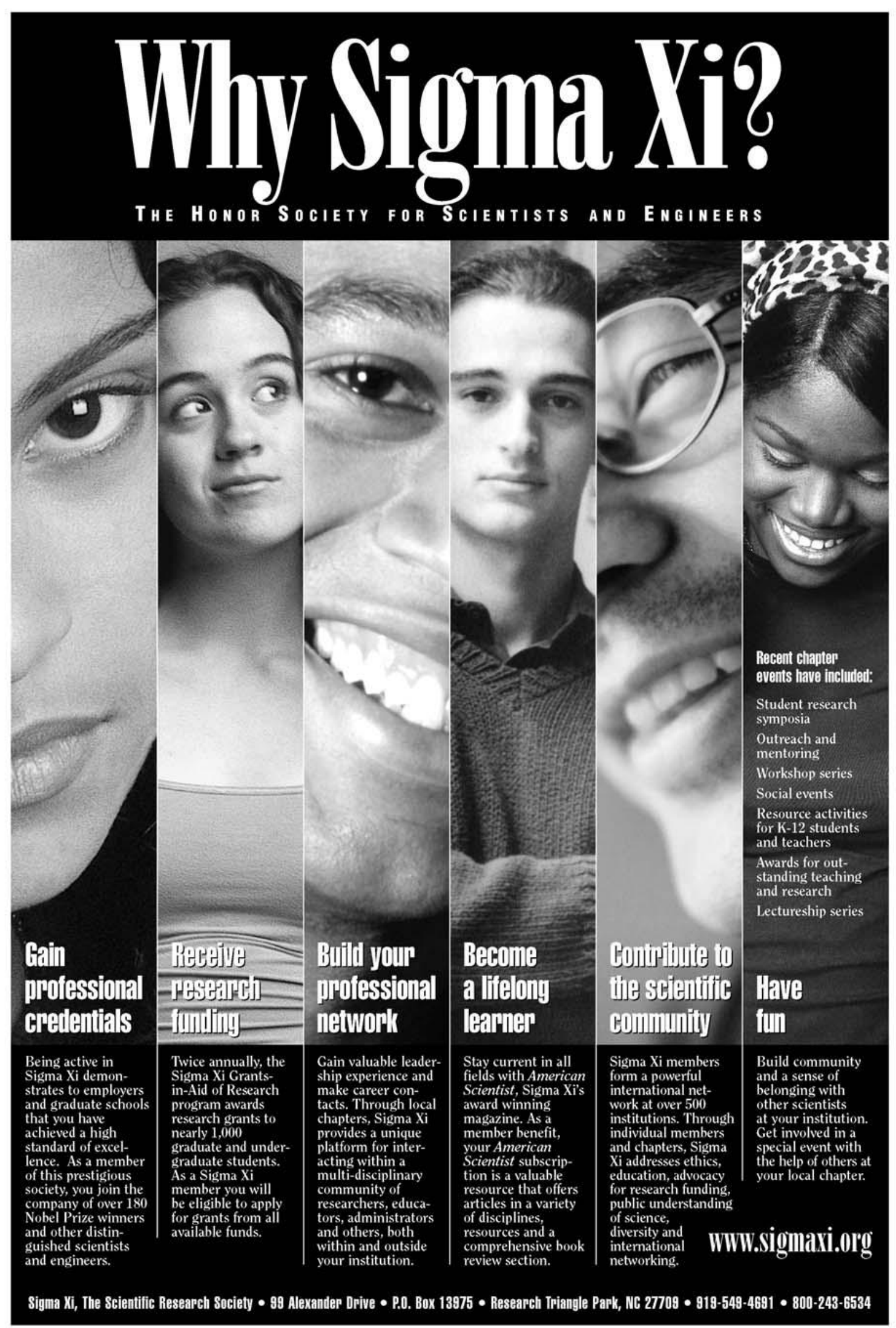




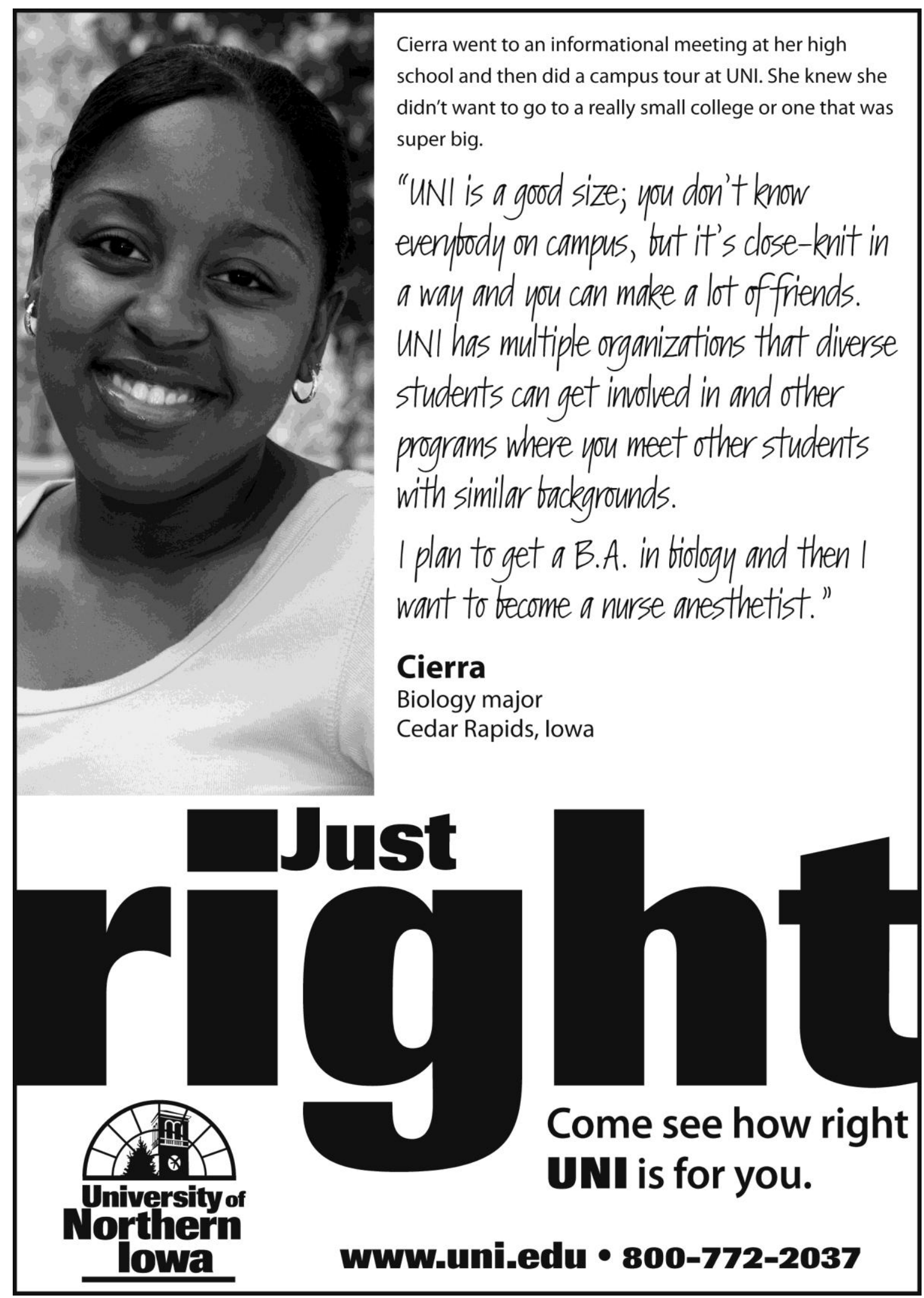

